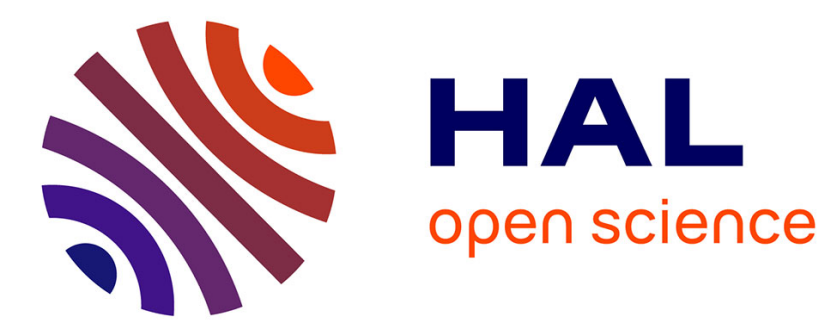

\title{
Path Planning of Unmanned Aerial Vehicles With Terrestrial Wireless Network Tracking
}

Mustapha Bekhti, Marwen Abdennebi, Nadjib Achir, Khaled Boussetta

\section{To cite this version:}

Mustapha Bekhti, Marwen Abdennebi, Nadjib Achir, Khaled Boussetta. Path Planning of Unmanned Aerial Vehicles With Terrestrial Wireless Network Tracking. Wireless days 2016, Mar 2016, Toulouse, France. 10.1109/WD.2016.7461521 . hal-01415634

\section{HAL Id: hal-01415634 https://sorbonne-paris-nord.hal.science/hal-01415634}

Submitted on 21 Jan 2017

HAL is a multi-disciplinary open access archive for the deposit and dissemination of scientific research documents, whether they are published or not. The documents may come from teaching and research institutions in France or abroad, or from public or private research centers.
L'archive ouverte pluridisciplinaire HAL, est destinée au dépôt et à la diffusion de documents scientifiques de niveau recherche, publiés ou non, émanant des établissements d'enseignement et de recherche français ou étrangers, des laboratoires publics ou privés. 


\section{PATH PLANNING OF UNMANNED AERIAL VEHICLES WITH TERRESTRIAL WIRELESS NETWORK TRACKING}

Mustapha Bekhti, Marwen Abdennebi, Nadjib Achir \& Khaled Boussetta

\section{CONTENTS}

1 Introduction

2 State of the art

3 Path Planning Problem Formulation

3.1 Problem statement and system description $\ldots \ldots \ldots . . . . .5$

3.2 Integer Linear Programming formulation . . . . . . . . . . . . . 6

3.3 Packet Loss Rate Estimation . . . . . . . . . . . . . . 7

4 Results

5 Conclusion

\section{LIST OF FIGURES}

Figure $1 \quad$ Discretization of the area $A$ into hexagonal Unit Areas 5 Figure $2 \quad$ Area Units $A U_{i}$ and its sub-areas $A_{k} \ldots \ldots$. Figure $3 \quad$ Shortest path Vs Obtained path with $\delta=0.1 \ldots 12$ Figure $4 \quad$ Shortest path Vs Obtained path with $\delta=0.9 \ldots \ldots \quad 12$ Figure 5 Path length Vs number of BS Vs Required accuracy . 13 Figure $6 \quad$ Percentage of admissible solutions ....... 13

LIST OF TABLES

Table 1

\section{ABSTRACT}

The UAVs (Unmanned Aerial Vehicles) market is projected to grow, sustained by the technological progress in different domains related to UAVs 
and by the emergence of new civilian applications. However, this economical development might be held back due to increased regulation constraints. A major concern of public authorities is to ensure a safe sharing of the airspace, especially over populated areas. To reach this aim, a fundamental mechanism is to provide a permanent tracking of UAVs. In this paper, we investigate the path planning of autonomous UAVs with tracking capabilities provided by terrestrial wireless networks. We formalize this problem as a constrained shortest path problem, where the objective is to minimize the delay for reaching a destination, while ensuring a certain delivery ratio of messages reporting the drone's positions.

* Université Paris 13, Sorbonne Paris Cité - L2TI (EA 4303) 99 Avenue J-B Clément, 93430 Villetaneuse, France\} 
Drones or UAVs (Unmanned Aerial Vehicles) are unmanned flying machines capable of carrying out more or less autonomous mission. Their earliest development was intended to the military use. Typical missions are the reconnaissance and the surveillance of wide and/or abroad territories. They are especially adapted for the realization of missions that would put the crew in danger or that would be tedious for an on-board crew. The technological progress in different domains that are related to UAVs, such as, advances in aeronautical, robotic, batteries and computer science have recently extended the economical perspectives toward the civil market. Beside entertainment, there are already several successful uses of UAVs for civil applications. One can refer to traffic monitoring in highways, prevention of forests fires, inspection of buildings and structures or data gathering for environment, for agriculture or for mining.

In addition, a forthcoming plans for freight and package delivery services has recently been announced by a number of companies around the world such as Deuch Post DHL, which has started testing the delivery of mails and medicament to hard-to-reach places, Amazon Inc for a UAV home package delivery program within the few next years or Zookal Inc, an Australian textbook rental company, which announced a program of delivering books to students in sight of cutting delivery times to minutes rather than days.

Unfortunately, even though UAVs are expected to revolutionize some civil applications, the legal and regulatory framework crawl behind. On the other side, from the commercial standpoint, the development of UAVs with higher level of autonomy and a minimum of persons controlling the drones actions are essential requirements to exploit this technology at its highest economical potential.

Thus, tracking is a fundamental mechanism that needs to be integrated into autonomous and even non-autonomous UAVs in order to provide a reliable system of collision avoidance that guarantees the safety of the overflown population.

While, this paper does not investigate the localization problem, which could for instance relays on classical outdoor localization solutions, like the GPS, we choose to focus on the transmission issue of the UAVs positions. The basic concept is to use terrestrial wireless networks, like cellular or WiFi technologies, to periodically transmit the UAVs' coordinates toward a remote monitoring and controlling system.

The main purpose of this work is to reshape the UAVs trajectory path depending not only on the destination but also on the capacity of the wireless networks (covering the target UAV area) to transmit the UAV localization messages with a satisfactory Quality of Service level. Precisely, we address the offline path planning of an UAV, which starting from a given position has to reach in a minimum possible delay a predetermined destination. The trajectory is constrained by the capacity of the drone to periodically transmit its localization to the remote system using traversed terrestrial wireless networks. The constraint is expressed as a maximum ratio of lost messages, which can occur either due to the wireless capacity limitations or due to the incomplete radio coverage of the traversed area.

The remainder of this paper is organized as follows. Section 2 briefly reviews related works. System description and formulation of the problem as well as the analytical model for computing the tracking packets loss rate are detailed in section 3. The performance evaluation results obtained af- 
ter solving the constrained optimization problem are analyzed in Section 4 . Finally, section 5 concludes the paper and discusses our future prospects.

\section{STATE OF THE ART}

Path planning issues have been widely studied in the robotics realm and have been addressed using different approaches and techniques. The two most popular techniques are deterministic, heuristic-based algorithms [8], [9], [10] and probabilistic, randomized algorithms [11] and [12]. The choice of the algorithm to use depends on the type of problem to be solved.

Thus we do not aim to provide an exhaustive list but we will be content to provide the most relevant work related to the path planning regarding to the nature of the objectives, problems formalization and resolving methods.

The authors in [2] mentioned that classical planning algorithms are based on different approaches such as geometric control [3], optimal control [4], flatness [5] stochastic theory[6]. On the other hand, the authors in [7] presented a survey of motion planning algorithms from the perspective of autonomous UAV guidance. They mentioned that guidance for fixed and rotary-wing UAV, involves significant differences from most traditionally mobile and manipulator robots. These includes three-dimensional environments, disturbed operating conditions, and high levels of uncertainty in state knowledge. To our knowledge, it does not exist an algorithm that provides a fully exact analytic solution to such a problem.

In [13], the authors presented a path planning for unmanned aerial vehicles in uncertain an adversarial environments in sight to reach a given target, while maximizing the safety of the drone. They proposed a path planning algorithm based on threats probability map, which can be built from a priori surveillance data. In the same context, [14] proposed an intelligent online path planning for UAVs in adversarial environments based on a model predictive control. Along the same lines, [15] considered the application of evolution-based path planning in the case of moving obstacles at uncertain locations. It was mentioned that changing the problem from fixed obstacles to moving ones, transform the problem from a geometric deterministic problem to a dynamic stochastic issue.

Finally, it is worth to mention the research done by [16] that can be considered one of the few papers dealing with path planning strategies destined for a based UAVs network. The authors compared deterministic and probabilistic path planning strategies for autonomous drones to explore a given area with obstacles and to provide an overview image. The results showed that although the deterministic approach could provide a solution it requires more knowledge and time to generate a plan. However, the probabilistic approaches are flexible and adaptive.

To the best of our knowledge, none of the above works have investigated UAV path planning problem assuming that UAV uses terrestrial wireless networks to transmit its locations. 


\section{PATH PLANNING PROBLEM FORMULATION}

\subsection{Problem statement and system description}

In this paper, we consider that an UAV starting from a given position $\mathrm{O}$ has to deliver a package to a predetermined destination, noted D. Our purpose is to provide an offline path planning with the objective of minimizing the delivery delay. The proposed path must be feasible with respect to the UAV's residual energy constraint. In addition, the path is computed offline with the aim of ensuring a quasi-permanent tracking of the UAV's location, using wireless networks technologies. For simplicity reasons we suppose that the drone keeps the same height from position $\mathrm{O}$ to destination $\mathrm{D} .{ }_{3} \mathrm{D}$ path planning is left for future investigations. The investigated geographical area is denoted $A$. Without a loss of generality, we assume that $A$ is a $2 D$ square area, which does not contain any obstacle.

Since discretization is necessary to reduce the set of possible paths from $\mathrm{O}$ to $\mathrm{D}$ to a finite dimension, $\mathrm{A}$ is discretized into $\mathrm{C}$ Area Units $(A U)$ of same hexagonal shape and same dimensions. The hexagonal shape is chosen to allow more flexibility in the drone's movement patterns. Furthermore,

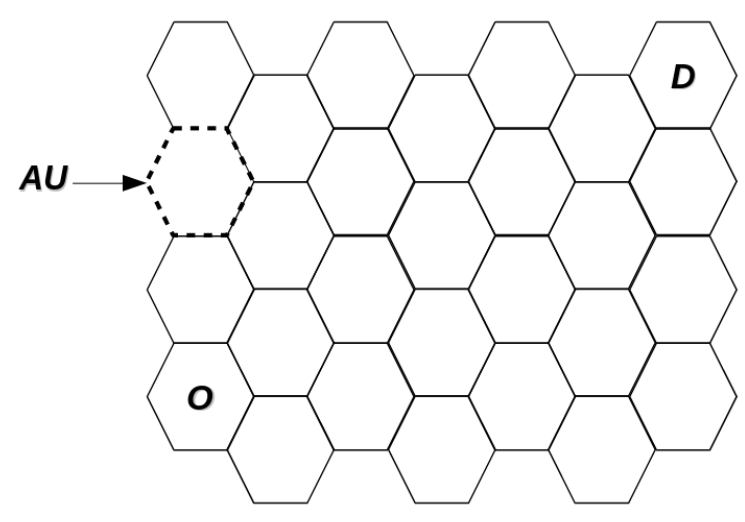

Figure 1: Discretization of the area $A$ into hexagonal Unit Areas

we suppose that an UAV flying over an $A U$ passes necessarily through its center. We thus choose to set $\mathrm{O}$ and $\mathrm{D}$ at the center of their associated $A U$ s, which we will refer to o and $d$, respectively. We also assume that the UAV trajectory between two adjacent $A U$ s follows the linear segment connecting their respective centers. Furthermore, we suppose that the UAVs velocity, denoted $S$, is constant along the path. Extension to path planning with dynamic velocity is left for future prospects. Our first goal is thus to determine the sequence of $A U$ s that must be traversed by a drone starting from o and going to $d$. The primary objective being to minimize the package delivery delay.

Another constraint that must be satisfied is the tracking of the drone's positions using wireless networks, such as cellular or IEEE 802.11x technologies. For this purpose, we assume that after each period $\mathrm{T}$ the drone generates a message of size $\mathrm{D}$ bits containing its most recent ${ }_{3} \mathrm{D}$ position. When possible, the on-board wireless interface tries to send each generated message to the remote UAV monitoring and controlling system. The opportunity to transmit depends on the radio coverage and the capacity of the related wireless technology in the drone's location. A message can also be corrupted due to radio transmission errors and discarded at the receiver 
side. We thus assume that a generated location message might be lost with a probability that we denote P. Our purpose is to provide a drone's path planning, which guarantee that the rate of successful transmissions of localization messages is above a given threshold denoted $\delta$. Formally, $1-\mathrm{P} \geqslant \delta$. The packet loss rate, $\mathrm{P}$, is computed as the proportion of lost messages over generated ones during the overall path. An analytic estimation of this metric depending on the location and the coverage of the wireless network is fully detailed in subsection 3.3.

In the following, we propose an Integer Linear Programming formulation of our path planning problem.

\subsection{Integer Linear Programming formulation}

Let $G=(V, E)$ be a directed graph. $V$ is the set of vertices and correspond to the set of $A U_{\mathrm{s}}$ in $\mathrm{A}$, and $\mathrm{E}$ is the set of edges and denotes the possible movements followed by any drone in order to move from one $A U$ to any another adjacent $A U$. Since we consider hexagonal shape for our $A U$ s thus only six possible movements are allowed. We also define a cost function matrix $C=\left(c_{i j}\right)$ denoting the cost for a drone when moving from vertex $i$ to vertex $j$, in its vicinity. We define $c_{i j}=d_{i j} / S$, where $d_{i j}$ is the distance required for traveling from $A U i$ to $A U j, i$ and $j \in V$ and $S$ is the linear speed of the drone. We also define $P_{i j}$ as the tracking packets loss probability when a drone is moving from $A U i \mathrm{i}$ to $A U \mathrm{j}$. According to the last considerations, our main problem is to:

- Minimize the UAV traveling delay between the origin vertix o and the destination vertix $d$.

- Guarantee that the rate of successful transmissions of localization messages over the selected UAV path is above a given threshold denoted $\delta$.

Given our system description and modeling, the above problem could be expressed a constrained shortest path problem using the following Integer Linear Programming formulation:

$$
\begin{array}{ll}
\min \quad & \sum_{i \in A} \sum_{j \in A} c_{i, j} x_{i j} \\
\text { s.t. } \quad & \sum_{j \in A} x_{o j}=1, \forall j \in A \\
& \sum_{i \in A} x_{i d}=1, \forall i \in A \\
& \sum_{i \in A} x_{i p}-\sum_{i \in A} x_{p j}=0, \forall i \in A ; \forall p \in A \\
& \sum_{i \in A} x_{i j} \leqslant 1, \forall j \in A \\
& \sum_{i \in A} \sum_{j \in A}\left(\left(1-P_{i j}\right)-\delta\right) x_{i j} \geqslant 0 \\
& x_{i j} \in\{0,1\}, \forall i, j \in A
\end{array}
$$

where $\mathrm{x}_{i, j}$ is a binary variable that is equal to 1 if the drone moves from $A U$ $i$ to $A U$ j, 0 otherwise. 
As indicated in the problem formulation, our objective (1a) is to minimize the travel time between the source and the destination. The first constraint (ib) states that the depart node of the drone is identified by the vertex o. As in the last constraint, (IC) guarantee that the destination of the drone is the vertex $\mathrm{d}$. The next constraint (Id) is for flow conservation. More precisely, this constraint guarantees that once a drone visits $A U i$, then it must also leave from this $A U$. The next constraint (1e) states that each $A U$ is visited only once. As introduced earlier, in addition to the last classical constraints of shortest path problem, we added a new constraint (If) which guarantees that the rate of successful transmissions of localization messages over the selected UAV path is above a given threshold denoted $\delta$.

\subsection{Packet Loss Rate Estimation}

In this section we evaluate the transmission capabilities of the radio interface according to the position of UAV and the wireless network stations (noted BS in the following) in the considered area. This evaluation is needed as it gives estimation for the achievable physical bitrate and for the packet loss rate, two parameters used for the optimization of the UAV flight plan in the area. For the achievable physical transmission rate, we need to determine, for each $A U$, the received power $\mathrm{P}_{\mathrm{r}}$ and the SINR (Signal to Noise/Interference ratio) in the BS side.

Assuming a transmission power $\mathrm{P}_{t}$ for the UAV, the received power $\mathrm{P}_{\mathrm{r}}$ is easily computed using an appropriate propagation model depending on the distance $d$ between the UAV and the BS. As we consider an open field area, this appropriate model could be either a two ray ground reflection or a free space, depending on the UAV altitude and the BS height connected to it. The selection between these two models is done according to the Fresnel zone, itself parametrized by the distance $d$ and the antenna heights, through the Fresnel ellipsoid radius $r$. In both cases, the propagation model consists of only the attenuation of the signal power that depends on the distance between UAV and the serving BS, as following:

$$
P_{r}=\operatorname{Att}(d) \cdot P_{t}
$$

In case of free space model, the attenuation $A_{t t} t_{F S}$ follows the Friis Formula and depends on the LoS (Line of Sight) distance between the transmitter (Tx) and the receiver (Rx) nodes, on the $T x / R x$ gains $G_{e}$ and $G_{r}$, and on the wavelength $\lambda$, as following:

$$
P_{r, F S}=A t t_{F S}(d) \cdot P_{t}=G_{e} G_{r}\left(\frac{\lambda}{4 \pi d}\right)^{2} P_{t}
$$

In case of a two ray ground model, the distance $\mathrm{d}^{*}$ used is the distance between the ground positions of the Tx/Rx nodes. The attenuation Att $\mathrm{TR}_{\mathrm{R}}$ is function of the ground reflection coefficient $\mathrm{L}$, and the $\mathrm{Tx} / \mathrm{Rx}$ antennas heights $H_{t}$ and $H_{r}$, as following:

$$
P_{r, T R}=\operatorname{Att}_{T R}(d) \cdot P_{t}=G_{e} G_{r} \cdot\left(H_{t} H_{r}\right)^{2}\left(\frac{L}{d^{*}}\right)^{4} P_{t}
$$

We should first determine the distances $d$ and $d^{*}$, depending on the UAV coordinates $(x, y, z)$ (its location in the $A U_{i}$ ) and on the coordinates $\left(X_{b s}, Y_{b s}, Z_{b s}\right)$ of the serving BS. This will be used to calculate the value of $r=\frac{1}{2} \sqrt{\lambda \cdot d^{*}}$ that will determine the choice of the appropriate model. 
As we consider a discrete area model, the received power for each $A U_{i}$ consists in averaging the received power $P_{r}(i)$ in the entire surface $S_{A U_{i}}$. Since we consider an hexagonal shapes for our area units, we can average the received power on each $A U_{i}$ by splitting the computation on each sub area $A_{0}, A_{1}, A_{2}, A_{3}$ and $A_{4}$, as illustrated in the figure 2. We can then

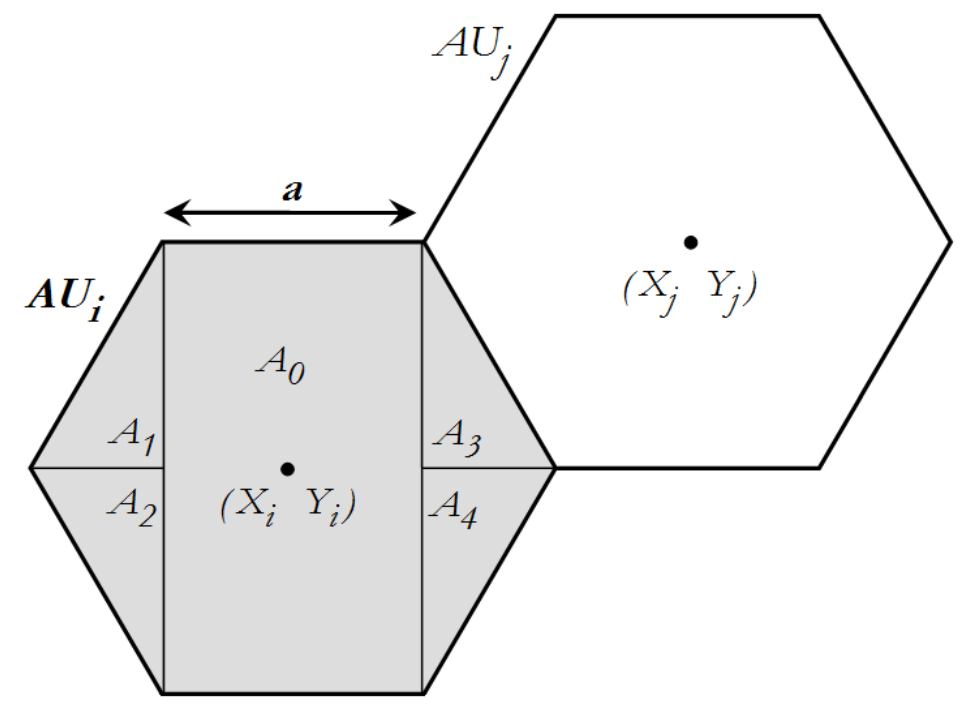

Figure 2: Area Units $A U_{i}$ and its sub-areas $A_{k}$

compute the average $P_{r}(i)$, for $A U_{i}$ assuming a constant $P_{t}$ (i.e. no power control) as following:

$$
P_{r}(i)=P_{t} \cdot \frac{1}{S_{A U_{i}}} \int_{S_{A U_{i}}} A t t\left(s\left(d_{i}\right)\right) \cdot d^{2} s
$$

After decomposition into the constant and the variable terms of the attenuation, $A_{c}$ and $A\left(s\left(d_{i}\right)\right)$ respectively, $P_{r}(i)$ becomes:

$$
\begin{array}{r}
P_{r}(i)=P_{t} \cdot A_{c} \cdot\left(\int_{S_{A_{0}}} A\left(s\left(d_{i}\right)\right) \cdot d^{2} s\right. \\
+\int_{S_{A_{1}}} A\left(s\left(d_{i}\right)\right) \cdot d^{2} s+\int_{S_{A_{2}}} A\left(s\left(d_{i}\right)\right) \cdot d^{2} s \\
\left.+\int_{S_{A_{3}}} A\left(s\left(d_{i}\right)\right) \cdot d^{2} s+\int_{S_{A_{4}}} A\left(s\left(d_{i}\right)\right) \cdot d^{2} s\right)
\end{array}
$$

Assuming a hexagon side length (or radius) of $a$, we give below an example for the average attenuation in each sub-area $A_{k}$. For sub-area $A_{0}$, it consists in a trivial calculation for the attenuation term:

$$
\int_{S_{A_{0}}} A\left(s\left(d_{i}\right)\right) \cdot d^{2} s=\int_{X i-l}^{X i+l} \int_{Y i-L}^{Y i+L} A(x, y) \cdot d y \cdot d x
$$

where $l=\frac{a}{2}$ and $L=a \frac{\sqrt{3}}{2}$. 
For the other terms (sub-areas $A_{k}, k \in\{1,2,3,4\}$ ), as the variables are inter-dependent, we should first determine the integration interval. We give an example for sub-area $A_{1}$.

$$
\begin{array}{r}
\int_{S_{A_{1}}} A\left(s\left(d_{i}\right)\right) \cdot d^{2} s=\int_{X i_{\text {min }}}^{X i_{\text {max }}} \int_{Y i_{\text {min }}}^{Y i_{\text {max }}} A(x, y) \cdot d y \cdot d x \\
=\int_{X i_{\text {min }}}^{X i_{\text {max }}} \int_{Y i_{\text {min }}}^{Y_{i}+\sqrt{3} \cdot\left(x-X_{i}-\frac{a}{2}\right)} A(x, y) \cdot d y \cdot d x
\end{array}
$$

where $X i_{\min }=X i-a ; X i_{\max }=X i-\frac{a}{2}$ and $Y i_{\min }=Y i$.

The constant value of $A_{c}$ and the variable $A\left(s\left(d_{i}\right)\right)$ depend on the selected propagation model. In Free Space environment $A_{\mathrm{c}_{\mathrm{FS}}}=\frac{\mathrm{Ge} \cdot \mathrm{Gr} \cdot \lambda^{2}}{16 \pi^{2} \cdot \mathrm{S}_{\mathrm{Au}}}=$ $\frac{\text { Ge.Gr. } \lambda^{2}}{8 \pi^{2} \cdot a^{2} \sqrt{3}}$, and $A\left(s\left(d_{i}\right)\right)$ is computed according to the coordinates $(x, y)$ of the UAV in the sub-area $A_{k}$ of $A U_{i}$ as following: $A(x, y)=A_{F S}(x, y)=$ $\left(\frac{1}{\sqrt{\left(x-X_{\mathrm{BS}}\right)^{2}+\left(y-Y_{\mathrm{BS}}\right)^{2}+\left(z-Z_{\mathrm{BS}}\right)^{2}}}\right)^{2}$. However, in the case of Two Ray ground environment $A_{\mathcal{C}_{T R}}=\frac{G e \cdot G r \cdot L^{4}\left(z \cdot Z_{B S}\right)}{S_{A U_{i}}}=\frac{2 \cdot G e \cdot G r \cdot L^{4}\left(z \cdot Z_{B S}\right)}{a^{2} \cdot \sqrt{3}}$ and $A(x, y)=$ $A_{\mathrm{TR}}(x, y)=\left(\frac{1}{\sqrt{\left(x-X_{\mathrm{BS}}\right)^{2}+\left(y-Y_{\mathrm{BS}}\right)^{2}}}\right)^{2}$.

Once $P_{r}(i)$ is determined, and assuming that the UAV connects to the BS that receives the highest power signal, the $\operatorname{SINR}(i)$ is computed for each $A U_{i}$ using the noise/interference power value. We then determine the MCS (Modulation and coding scheme) that should be selected by the AMC and which gives the transmission rate Rate $(i)$ in considered $A U_{i}$. In this case, the $\operatorname{SINR}(i)$ is equal to:

$$
\operatorname{SINR}(\mathfrak{i})=10 \cdot \log \left(\frac{\mathrm{P}_{\mathrm{r}}(\mathfrak{i})}{\mathrm{P}_{\text {Noise }}(\mathfrak{i})+\mathrm{P}_{\text {Interf }}(\mathfrak{i})}\right)
$$

The next step is to derive transmission error rate for each frame and in each Area Unit. This value will give us the link reliability in each $A U_{i}$ and hence for each transition $A U_{i} \rightarrow A U_{j}$. In each $A U_{i}$, an $\operatorname{SINR}(i)$ has been computed and is used to compute the ratio of energy per symbol to noise, $\mathrm{Es} / \mathrm{NO}$ with $\mathrm{E}_{s} / \mathrm{N}_{\mathrm{O}}=\mathrm{SINR} \cdot \frac{\mathrm{B}}{\mathrm{R}}$, with $\mathrm{R}$ and $\mathrm{B}$ the symbol rate and the channel bandwidth respectively.

Assuming that noise and interference can be considered as a whole as an additive white gaussian noise (AWGN) we then compute symbol error rate $P_{\text {sym }}$ using the well known complementary error function erfc which gives the probability of exceeding a threshold $x$.

For BPSK for example, we have this well known expression based on energies ratios $E_{s} / N_{0}$ :

$$
P_{s y m}=\frac{1}{2} \cdot \operatorname{erfc}\left(\sqrt{\frac{E_{s}}{N_{0}}}\right)
$$

For M-QAM ( $\mathrm{M}=4$ gives QPSK), we use the following equation as explained in [17]:

$$
\mathrm{P}_{\mathrm{sym}}=1-\left(1-\left(1-\frac{1}{\sqrt{M}}\right) \cdot \operatorname{erfc}\left(\sqrt{\frac{3 \cdot \mathrm{E}_{\mathrm{s}}}{2(M-1) \cdot \mathrm{N}_{0}}}\right)\right)^{2}
$$

Assuming a Gray coding, we then compute the bit error rate (BER) or probability $\mathrm{P}_{\mathrm{bit}}$, depending on the coding scheme. 
where $M$ is the number of symbol in the constellation of the considered modulation scheme

Once the BER $\mathrm{P}_{\mathrm{bit}}$ is computed, we can deduce transmission success probability $\mathrm{P}_{\text {succ }}$ of each frame according to the turbo code hamming distance which gives the maximum acceptable number of erroneous bits $N_{\text {berr }}$ per frame.

Assuming an MPDU size of D and a coding rate of $C_{R}$, the hamming distance $D_{\text {hamm }}$ for LDPC turbo codes used in WiFi network is given by: $D_{\text {hamm }}=n-k+1$, with $n=\frac{k}{C_{R}}, k=D$ and $N_{\text {berr }}=\frac{D_{\text {hamm }}-1}{2}=\frac{n-k}{2}$. Hence the $P_{\text {succ }}$ is computed as following:

$$
P_{\text {succ }}=\sum_{b=0}^{\frac{n-k}{2}}\left(\begin{array}{l}
n \\
b
\end{array}\right) \cdot B E R^{b} \cdot(1-B E R)^{(n-b)}
$$

Then, the mean number of frames received successfully.

$$
E\left[N_{t}\right]=\sum_{t=0}^{N_{\max }} t \cdot\left(\begin{array}{c}
N_{\max } \\
t
\end{array}\right) \cdot(1-P)^{t} \cdot P^{N_{\max }-t}
$$

where $\mathrm{N}_{\max }$ is the maximum number of transmitted frames.

We deduce the mean number of frame received successfully for an $A U_{i} \rightarrow$ $A U_{j}$ transition which is the sum for each $A U$ and for each mean number of frames $N_{\max }(i)$ sent in the considered $A U_{i}$. Note that as we suppose no MAC retransmission (retry limit set to o), the packet loss rate will be equal to the frame loss rate.

$$
E\left[N_{i j}\right]=E\left[N_{t}\left(N_{\max , i}\right)\right]+E\left[N_{t}\left(N_{\max , j}\right)\right]
$$

$N_{\max }(i)$ in each $A U_{i}$ depends on the packets rate (PckRate) generated and on the mean sojourn time in the considered $A U_{i}$. The sojourn time itself depends on the distance traveled in each $A U_{i}$ and on the UAV velocity S.

$$
\mathrm{N}_{\text {max }, i}=\operatorname{PckRate} \cdot \frac{\operatorname{dist}\left(\mathrm{AU}_{\mathrm{i}}\right)}{\mathrm{S}}
$$

We then compute the loss rate $P_{i j}$ for each transition $A U_{i} \rightarrow A U_{j} . P_{i j}$ is needed in equation (If) and is deduced from loss rate in each considered $A U_{i}, P_{i}=1-P_{\operatorname{succ}}(i)$ :

$$
P_{i j}=\frac{N_{\max , i} P_{i}+N_{\max , j} P_{j}}{N_{\max , i}+N_{\max , j}}
$$

\section{RESULTS}

In this section, we analyze the performances of our proposed path planning algorithm. We consider a scenario with an area size of $250 \mathrm{~m} \times 250 \mathrm{~m}$. We vary the number of BS from 5 to 35 BSs with random positions. We consider the antennas gains as constant. Despite the UAV mobility and the use of omnidirectionnal antennas, this assumption is reasonable as we consider small difference between Tx and Rx heights (UAV and BSs). Moreover, as we consider constant AUV velocity $S$ and uniform trajectories in each Area Unit, we can fix the distance traveled in each $\mathrm{AU}$ and hence the number of transmit packets $N_{\max }$, i.e., $\mathrm{N}_{\max , i}=\mathrm{N}_{\max , j} \forall i, j \in A$. We also run 20 times each simulation. Table 1 summarizes all our pre-defined parameters. 
For our simulations, we use MATLAB in order to compute the packet loss probability $\mathrm{P}_{i j}$ and in order to resolve our optimization problem we used CPLEX.

Table 1: Parameters configuration

\begin{tabular}{|l|l|}
\hline $\mathrm{D}$ & 200 bytes \\
\hline $\mathrm{P}_{\mathrm{t}}$ & 20 dBm (100 $\mathrm{mW})$ \\
\hline Pnoise + Pinterf & $-60 \mathrm{dBm}($ Constant) \\
\hline Antennas Gains & $\mathrm{Ge}=\mathrm{Gr}=10 \mathrm{dBi}$ \\
\hline AU radius (constant) & $\mathrm{a}=5 \mathrm{~m}$ \\
\hline Area & $\mathrm{X}=\mathrm{Y}=250 \mathrm{~m}$ \\
\hline Carrier Frequency & $2.4 \mathrm{GHz}$ \\
\hline UAV altitude & Fixed, $\mathrm{z}=10 \mathrm{~m}=\mathrm{Ht}$ \\
\hline BSs & from 5 to 35 \\
\hline$\delta$ & from 0.1 to 0.9 \\
\hline
\end{tabular}

In figure ?? we show an example of the perceived SINR where we randomly deploy a wireless network with 25 base stations. As we can see, some area units have good SINR while others offer a bad SINR. In this case, it is more judicious for drones to follow a trajectory that maximize the tracking process by maximizing the packet tracking delivery along this trajectory. Note that we considered an AMC function parameters as designed in [18]. In order to illustrate our proposal, we plot in figures 3 and 4 the obtained path compared to the shortest path (using Dijkstra's algorithm) for $\delta=0.1$ and $\delta=0.9$ respectively. As we can see in 3 , when we fix the delivery threshold to $\delta=0.1$ the obtained path, in yellow, is very close the shortest path and has exactly the same length. However, we can see in figure 4 that if we increase the tracking accuracy $(\delta=0.9)$ we obtain a more complex path and much longer than the shortest path. We can also notice that the followed path is matching the positions of the deployed base stations.

In figure 5, we plot the path length of the obtained path when varying the number of deployed wireless base stations (from 5 to 35) and for different tracking accuracy $(\delta=0.2, \delta=0.4, \delta=0.6, \delta=0.8)$. We can clearly notice that more we increase the tracking accuracy longer is the path. This observation is expected, since increasing the accuracy lead to more winding path in order to guarantee this accuracy on the overall path. However, when we increase the number of deployed base stations the path length tends to be reduced since we are increasing the possible solutions.

Finally, in figure 6, we plot the percentage of time where the CPLEX solver was able to find a solution for a given number of base stations and a given tracking accuracy. Our constrained optimization problem is unfeasible when both the current positions of the base stations as well as the required accuracy are too strong to find a path between the source and the destination. As we can see, it is almost always possible to find a solution if the tracking accuracy is reduced (small values of $\delta$ ). However, when we increase the accuracy while reducing the number of base stations, the number of obtained solutions to our optimization problem decreases.

\section{CONCLUSION}

Tracking is a fundamental mechanism that needs to be integrated into UAVs in order to enforce safety. To the best of our knowledge, this work is the first one to propose a path planning of UAV with the aim of minimizing the delay to reach a destination, while ensuring that the UAV is able to 


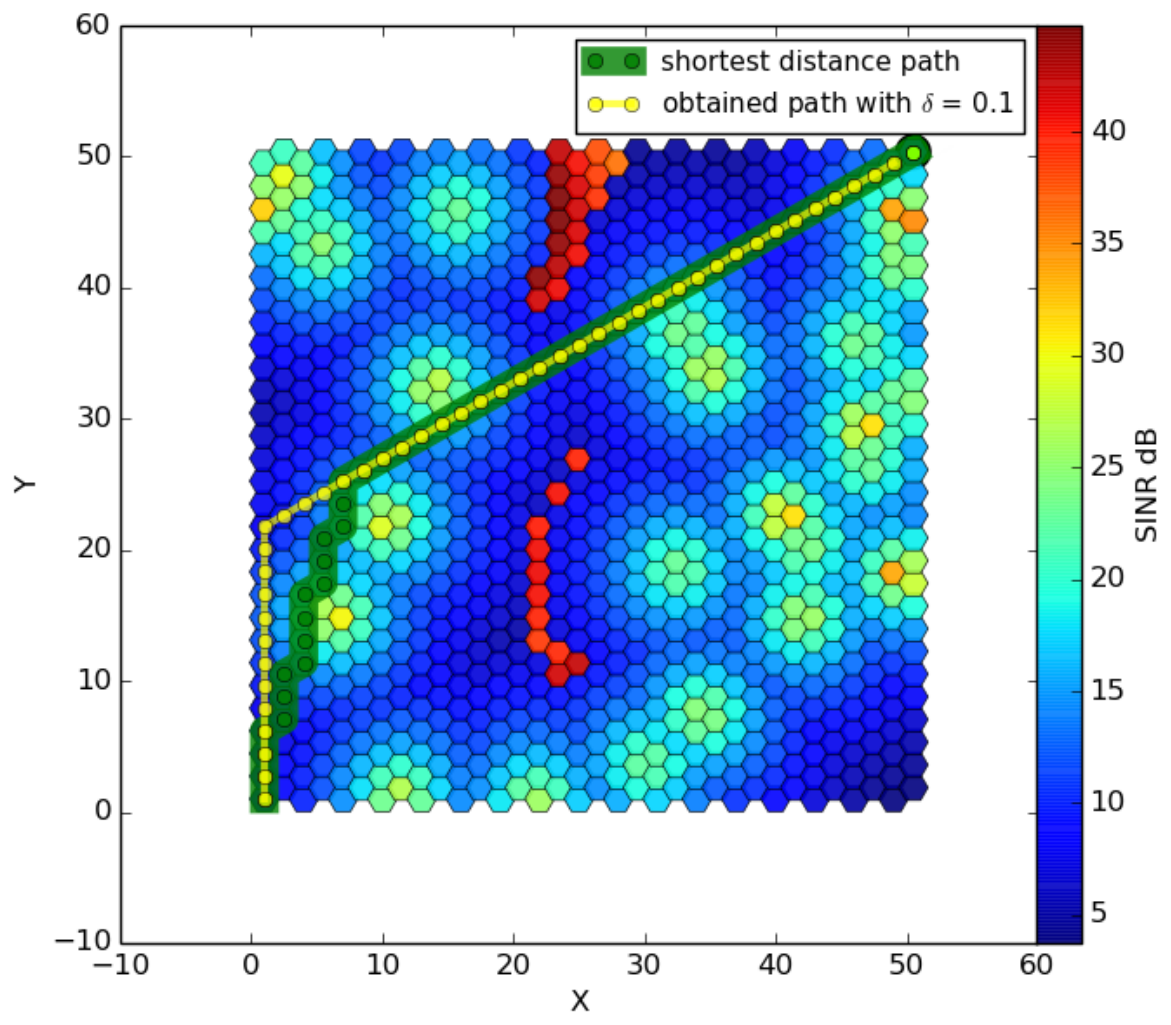

Figure 3: Shortest path Vs Obtained path with $\delta=0.1$

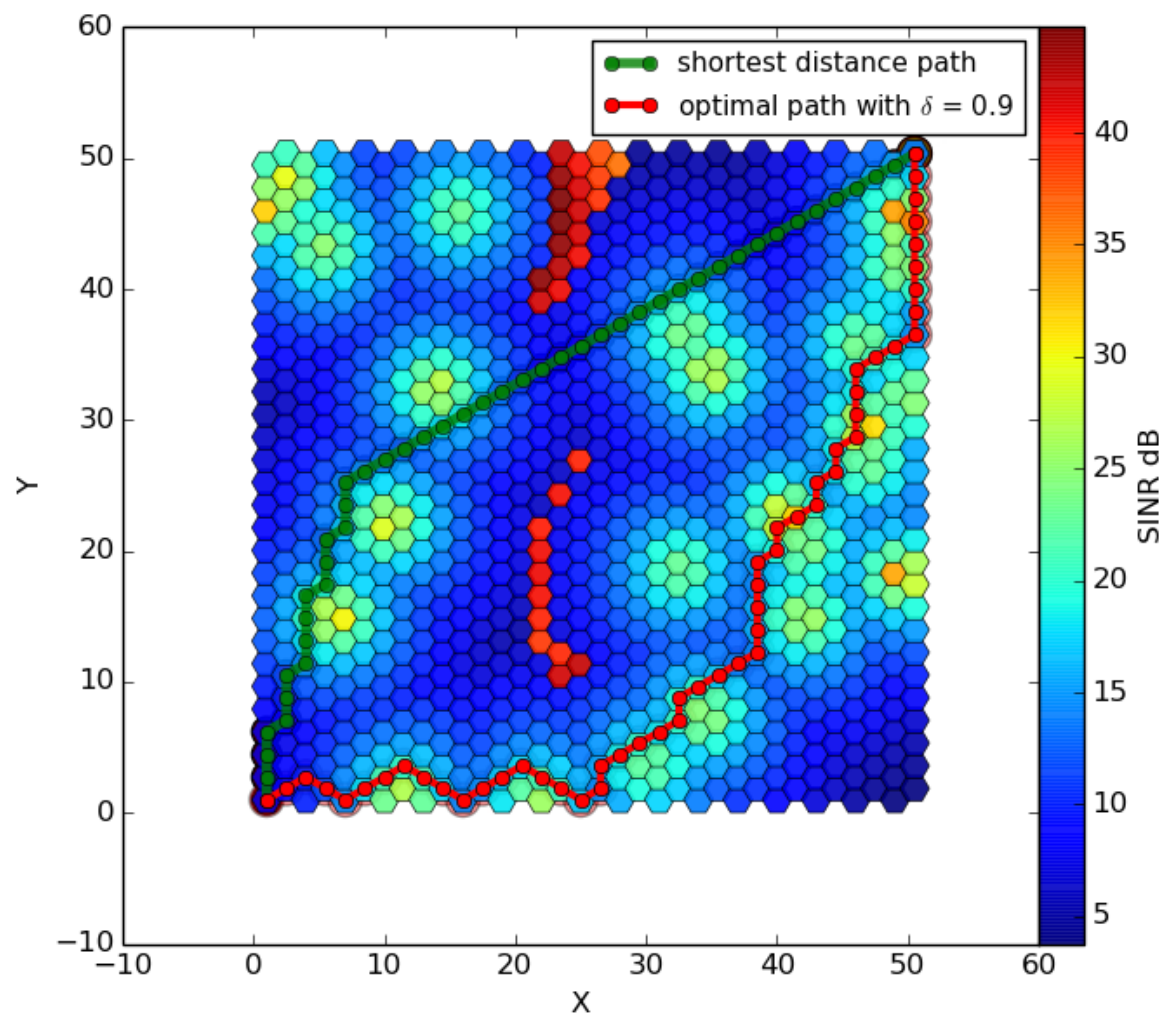

Figure 4: Shortest path Vs Obtained path with $\delta=0.9$ 


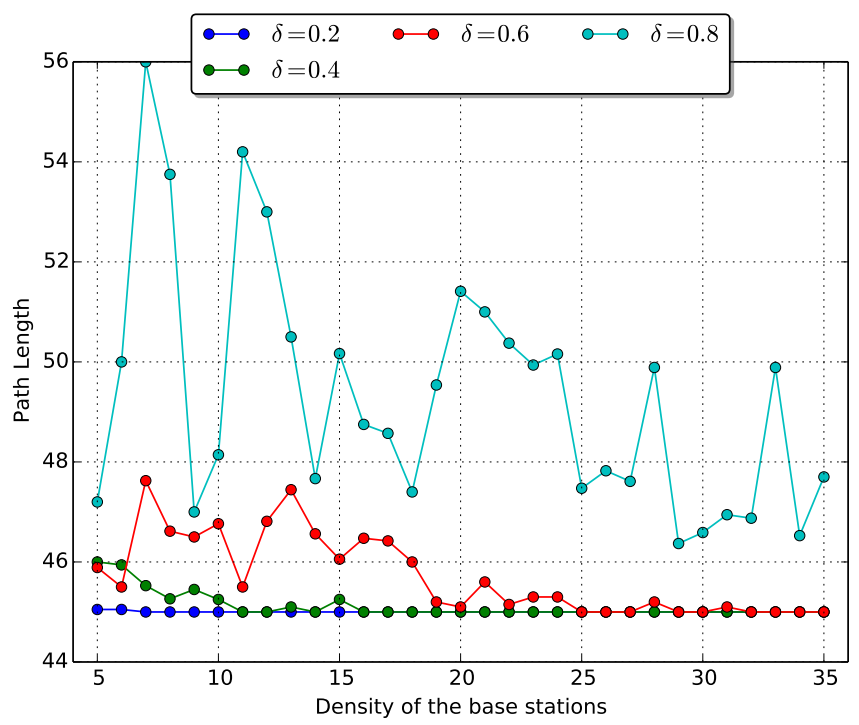

Figure 5: Path length Vs number of BS Vs Required accuracy

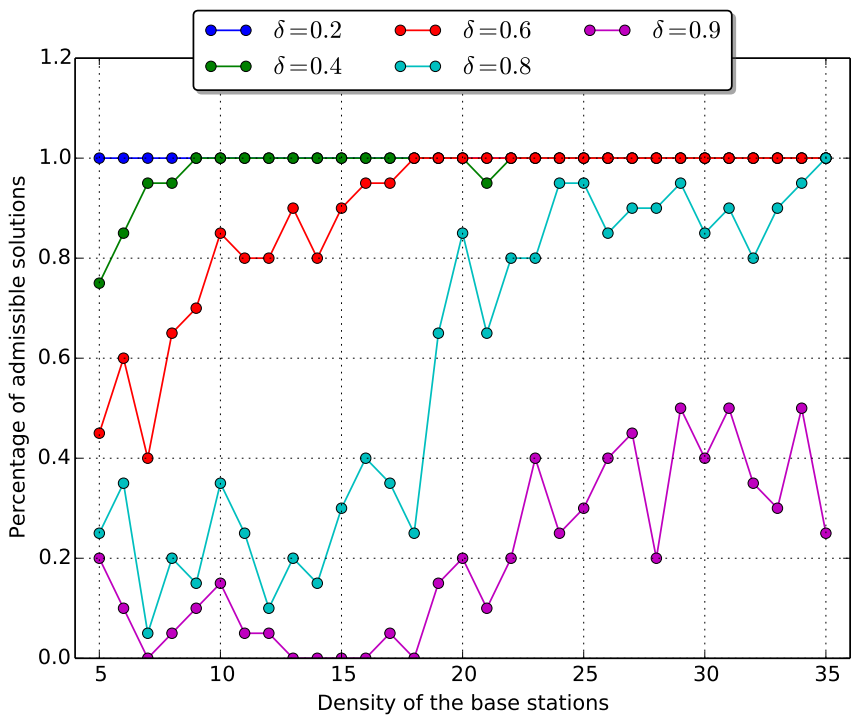

Figure 6: Percentage of admissible solutions

transmit periodically its positions using terrestrial wireless networks, with a maximum threshold on packets losses. We formulate the above problem as an Integer Linear Problem. To this purpose we also express analytically the packet loss rate of tracking messages depending on the UAV location and the wireless network coverage. Solving the ILP problem using CPLEX, we were able to analyze how the radio coverage (i.e. density of BS) as well as the threshold on the packet success rate, impact the number of possible solutions and the trajectory of the UAV. Our current investigations focus on the complexity issue raised for larger size of the area $A$. We are currently designing heuristics to cope with the curse of dimensionality. Related to this issue, we are also extending our problem to the ${ }_{3} \mathrm{D}$ case. Finally, we are also focusing on modeling the energy consumption of the UAV, depending on its 
trajectory, weight, altitude and communications, with the aim to integrate the energy autonomy as an additional constraints.

\section{REFERENCES}

[1] Wang, Honglun, et al. "Three-dimensional path planning for unmanned aerial vehicle based on interfered fluid dynamical system." Chinese Journal of Aeronautics 28.1 (2015): 229-239.

[2] Maillot, Thibault, et al. "Lyapunov and minimum-time path planning for drones." Journal of Dynamical and Control Systems 21.1 (2015): 47-80.

[3] Cichella, Venanzio, et al. "Geometric $3 \mathrm{D}$ path-following control for a fixed-wing UAV on SO (3)." AIAA Conference of Guidance, Navigation and Control conference. 2011.

[4] Ding, Xu Chu, Amir R. Rahmani, and Magnus Egerstedt. "Multi-UAV convoy protection: an optimal approach to path planning and coordination." Robotics, IEEE Transactions on 26.2 (2010): 256-268.

[5] Bhatia, Amit, et al. "Dubins trajectory tracking using commercial off-theshelf autopilots." AIAA Guidance, Navigation, and Control Conference, Honolulu, Hawaii. 2008.

[6] Bertuccelli, Luca F., Albert Wu, and Jonathan P. How. "Robust adaptive Markov decision processes: planning with model uncertainty." Control Systems, IEEE 32.5 (2012): 96-109.

[7] Goerzen, C., Zhaodan Kong, and Bernard Mettler. "A survey of motion planning algorithms from the perspective of autonomous UAV guidance." Journal of Intelligent and Robotic Systems 57.1-4 (2010): 65-100.

[8] Hart, Peter E., Nils J. Nilsson, and Bertram Raphael. "A formal basis for the heuristic determination of minimum cost paths." Systems Science and Cybernetics, IEEE Transactions on 4.2 (1968): 100-107.

[9] Nilsson, Nils J. Principles of artificial intelligence. Morgan Kaufmann, 2014.

[10] Podsedkowski, Leszek, et al. "A new solution for path planning in partially known or unknown environment for nonholonomic mobile robots." Robotics and Autonomous Systems 34.2 (2001): 145-152.

[11] Kavraki, Lydia E., et al. "Probabilistic roadmaps for path planning in high-dimensional configuration spaces." Robotics and Automation, IEEE Transactions on 12.4 (1996): 566-580.

[12] LaValle, Steven M., and James J. Kuffner. "Randomized kinodynamic planning." The International Journal of Robotics Research 20.5 (2001): 378-400.

[13] Jun, Myungsoo, and Raffaello D’Andrea. "Path planning for unmanned aerial vehicles in uncertain and adversarial environments." Cooperative Control: Models, Applications and Algorithms. Springer US, 2003. 95110.

[14] Peng, Xingguang, and Demin Xu. "Intelligent online path planning for UAVs in adversarial environments." Int J Adv Rob Syst 9 (2012): 1-12. 
[15] Rathbun, David, et al. "An evolution based path planning algorithm for autonomous motion of a UAV through uncertain environments." Digital Avionics Systems Conference, 2002. Proceedings. The 21st. Vol. 2. IEEE, 2002.

[16] Yanmaz, Evşen, et al. "On path planning strategies for networked unmanned aerial vehicles." Computer Communications Workshops (INFOCOM WKSHPS), 2011 IEEE Conference on. IEEE, 2011.

[17] John G. Proakis, Digital Communications, 4th ed. McGraw-Hill Series in Electrical and Computer Engineering. Computer Engineering, 2001.

[18] Cisco Design Considerations, Wireless Mesh Access Points, Design and Deployment Guide, Release 7.3, Cisco 